\title{
Oral Benign Fibrous Histiocytoma - A Review of Literature from 1964-2016
}

\author{
Prasanna Kumar* \\ Rajiv Gandhi University of Health Sciences, India
}

Submission: March 18, 2017; Published: May 17, 2018

*Corresponding author: Prasanna Kumar, Rajiv Gandhi University of Health Sciences, Dept of Oral \& MAxillofacial Surgery, Sullia, Bangalore, India, Tel: 9448177525; Email: dr_prasanna_74@yahoo.co.in

\section{Introduction}

It all began way before the 1960's, however on one fateful day in the year of 1961, Kauffman ST and Stout AP changed the way the world looked at fibrous soft tissue tumours by being the first to report about Fibrous Histiocytoma and recognising it as a separate clinical entity [1].

Benign fibrous histiocytoma designates a group of quasineoplastic lesions that show both fibroblastic and histiocytic differentiation. Whether the lesions originate from histiocytic or fibroblastic tissues has not been clearly determined yet [2].

Some experts hypothesize that the cells originate from the tissue histiocytes and then assume fibroblastic properties [3] while others argue that immunohistochemical evidence of factor XII a positivity favours a dermal dendrocytic cell origin [4]. In consequence of the controversies of origin, over the years, BFH has been designatedby several names and classifications, such as sclerosing hemangioma, hemangioma cutis, fibroxanthoma and nodular subepidermal fibrosis [3].

$\mathrm{BFH}$ can be cutaneous and Non-cutaneous in nature. Cutaneous BFH commonly originates in sun exposed skin. Non cutaneous BFH represents approximately $1 \%$ of all benign $\mathrm{FH}$ lesions and most frequently occurs in soft tissues in the lower extremities(50\%), less frequently in the upper extremities (20\%), retroperitoneum (20\%) [5].

Benign FH can be categorised into superficial and deep forma. Deep benign FH is very rare, comprising less than 5\% [6] of all benign FH tumours. Fibrous Histiocytoma as reported in literature can present as malignant fibrous histiocytoma or benign Fibrous histiocytoma and may involve soft tissue as well as hard tissue [1]

The incidence of BFH in the oral cavity is rare, with few reported cases in the buccal spaces, tongue, gingival or alveolar ridge, mandible, maxilla, lower and upper lip, soft palate and floor of the mouth have also been described. Rare occurrences also include nasal cavity and paranasal sinuses, larynx, trachea, temporomandibular joint and submandibular and parotid glands $[5,7]$. The aim of this article is to trace the behaviour of Oral Benign Fibrous Histiocytoma across the literature and discuss the diagnostic techniques, current protocols in treatment and incidence of metastasis or recurrences if any.

\section{Discussion}

The oral and perioral cases of BFH, Gray et al. [8] found that the mean age of patients was 55 years ranging from 12 to 71 years. [9] Women are more frequently affected than men. Bielamowicz et al. [9] in their study of BFH in the head and neck region found M: F ratio of 2.5:1 [10].

The clinical picture as seen in literature varies significantly depending upon the location, duration and possible aetiology. Various causes have been speculated in the aetiology of BFH namely secondary to trauma, infection even immuno-suppression in some cases [11].

Clinically these benign tumours can present as asymptomatic [5], solitary, gradually enlarging growth that is well-circumscribed, painless and does not show aggressive behaviour or damage overlying mucosa. The most common chief complaint a patient presents with is a swelling with possible facial asymmetry and in some cases pain [1].

In rare conditions a patient may complain of nasal obstruction, nasal discharge, and episodes of epistaxis in case of involvement of the maxilla.[maxilla] or dysphagia, dyspnoea and difficulty in speech if present over the lingual or palatine region $[9,12]$.

On oral examination, it can present as an elastic soft or firm -elastic in consistency [9], demarcated and painless mass [upper lip] with no ulceration or involvement of adjacent structures.

The diagnosis and analysis can be challenging and is usually based on a combination of histopathology, light microscopy and immunohistochemistry [5].

\section{Histiology}

The histopathological picture usually show a non-infiltrating fibro histiocytic lesion composed of interlacing fascicles of spindle cells having plump and vesicular nucleus with tapered and blunt ends arranged in a typical storiform pattern. [7], 
densely proliferated histiocytes, spindle shaped tumour cells [8] or round histiocyte-like cells, lipid-containing xanthoma cells, multinucleated giant cells, and scattered lymphocytes are a frequent finding [7] (Table 1).

Table 1: Histiogenesis of BFH.

\begin{tabular}{|c|c|}
\hline Evidence in support of histiocytic origin & Evidence in support of fibroblastic origin \\
\hline Presence of lysosomal and proteolytic enzymes & Appearance of the lesional cells resembles fibroblast histologically \\
\hline Lesional cells exhibit phagocytic activity & \multirow{3}{*}{ Lack of expression of histiocytic marker (Langerhans granules) } \\
\hline Cells contain lipid & \\
\hline Multinucleated osteoclasts like cells present & \\
\hline
\end{tabular}

The differentiation between $\mathrm{BFH}$ and $\mathrm{MFH}$ on a histopathological picture can be made in the absence of cellular atypia [9], high mitotic activity, high pleomorphism of cells [12], hyperchromasia, atypicality of the nucleus and nuclear fission [8] which are characteristic features of MFH.

Table 2: Stainability of immunohistochemical staining

\begin{tabular}{|c|c|}
\hline Search Antibody & Stainability \\
\hline S-100 & - \\
\hline NSE & - \\
\hline$\alpha 1$-ACT & + \\
\hline Lysozyme & + \\
\hline CD68 & + \\
\hline Vimentin & + \\
\hline
\end{tabular}

-: No stain + : positive

Due to the lack of specific markers for fibrohistiocytic lesions, the diagnosis of BFH is generally based on the absence of markers for cells of other lineages [10]. The immunohistochemistry diagnosis is carried out formalin fixed, paraffin-embedded sections using streptavidin-biotin-peroxidase complex labelling method can be used. BFH shows immunostaining for vimentin $(+)$, CD68 (+), CD34 (+), S100 (-), CD117 (-), Leu7 (-), desmin (-), and $\alpha$ - SMA (-) $[10,13]$ (Table 2).

CT scans can be of diagnostic aid in Fibrohistiocytic tumours of the bone which presents as a well defined, expansile lytic lesion may or may not be associated with thinning or breach in the cortical plates.MRI scans are used in case of Fibrohistiocytic tumours of the soft tissues which show up as heterogeneously hyper intense on T2-weighted image [14-16]. Role of PET scans is not much tapped into and may pave way for better imaging in the recent future.

There seems to be a consensus across literature on the treatment protocol of Benign Fibrous Histiocytoma. The treatment is surgical en-bloc resection of the tumour with a safe margin of $5 \mathrm{~mm}$ and regular follow-up upto 3 years. $\mathrm{BFH}$ has a malignant form, which is more often encountered in the literature, Malignant Fibrous Histiocytomaand is described as having a local aggressiveness and a low rate of metastasis $[12,17]$. MFH is a primitive, pleomorphic sarcoma consisting partly of fibroblastic cells and partly histiocytic cells. Reported incidence of BFH to malignant transformation is $1 \%$ [18].

MFH has been an enigma since no true cell origin has been determined. WHO declassified MFH as a formal diagnostic entity and renamed it as undifferentiated pleomorphic sarcoma (WHOCT 2002).

The prognosis of oral BFH is usually very good. A rare case of metastasis has been documented with the angiomatoid variant of Oral BFH [17].

There also is a case report of a malignant transformation of oral benign Fibrous Histiocytoma lesion which was treated with aggressive surgical management and chemo/radiotherapy [1822].

Table 3: Review of cases of BFH of Soft tissues in chronological order.

\begin{tabular}{|c|c|c|c|c|c|c|}
\hline No. of cases & Authors & Age/Sex & Location & Treatment & $\begin{array}{c}\text { FU time/ } \\
\text { Recurrence }\end{array}$ & Year \\
\hline 1 & Prisse et al. [2] & $48 / F$ & Lower lip & SE & 7M/NED & 2015 \\
\hline 1 & Prisse et al. [2] & $75 / M$ & Palate & SE & 14M/NED & 2015 \\
\hline 1 & Prisse et al. [2] & $81 / \mathrm{M}$ & $\begin{array}{l}\text { Soft and Hard } \\
\text { Palate Junction }\end{array}$ & SE & 18M/NED & 2015 \\
\hline 1 & Giovani et al. [5] & $36 / \mathrm{M}$ & Buccal Mucosa & SE & 12M/NED & 2010 \\
\hline
\end{tabular}


Advances in Dentistry \& Oral Health

\begin{tabular}{|c|c|c|c|c|c|c|}
\hline 1 & Eu Jo et al. [6] & $36 / F$ & Buccal Mucosa & $\mathrm{SE}$ & 7M/NED & 2015 \\
\hline 1 & Femiano et al. [7] & $32 / \mathrm{M}$ & Buccal Mucosa & SE & $*$ & 2001 \\
\hline 1 & George et al. [7] & $37 / \mathrm{F}$ & Maxillary Gingiva & SE & 18M/NED & 2014 \\
\hline 1 & Gray et al. [8] & $45 / \mathrm{M}$ & Upper Lip & SE & & 1992 \\
\hline 1 & Gray et al. [8] & $42 / \mathrm{M}$ & Buccal Mucosa & SE & & 1992 \\
\hline 1 & Gray et al. [8] & $65 / \mathrm{M}$ & Buccal Mucosa & SE & & 1992 \\
\hline 1 & Gray et al. [8] & $37 / \mathrm{F}$ & Tongue & SE & & 1992 \\
\hline 1 & Gray et al. [8] & $50 / \mathrm{F}$ & Dorsum of Tongue & $\mathrm{SE}$ & & 1992 \\
\hline 1 & Gray et al. [8] & $71 / \mathrm{F}$ & Buccal Mucosa & SE & & 1992 \\
\hline 1 & Gray et al. [8] & $45 / F$ & Lower lip & SE & & 1992 \\
\hline 1 & Gray et al. [8] & $49 / \mathrm{M}$ & Maxillary Vestibule & $\mathrm{SE}$ & & 1992 \\
\hline 1 & Gray et al. [8] & $70 / F$ & Buccal Mucosa & SE & & 1992 \\
\hline 1 & Gray et al. [8] & $60 / \mathrm{M}$ & $\begin{array}{l}\text { Mandibular } \\
\text { Vestibule }\end{array}$ & SE & & 1992 \\
\hline 1 & Gray et al. [8] & $68 / F$ & Buccal Mucosa & SE & & 1992 \\
\hline 1 & Gray et al. [8] & & $\begin{array}{l}\text { Mandibular } \\
\text { Vestibule }\end{array}$ & $\mathrm{SE}$ & & 1992 \\
\hline 1 & Gray et al. [8] & $66 / F$ & $\begin{array}{l}\text { Mandibular } \\
\text { Vestibule }\end{array}$ & SE & & 1992 \\
\hline 1 & Gray et al. [8] & $37 / \mathrm{F}$ & Maxillary Gingiva & SE & & 1992 \\
\hline 1 & $\begin{array}{l}\text { Bielamowicz et } \\
\text { al. [9] }\end{array}$ & $25 / \mathrm{M}$ & Buccal Mucosa & $\mathrm{SE}$ & 24M/NED & 1995 \\
\hline 1 & $\begin{array}{l}\text { Bielamowicz et } \\
\text { al. [9] }\end{array}$ & $49 / \mathrm{M}$ & $\begin{array}{c}\text { Submandibular } \\
\text { Region }\end{array}$ & $\mathrm{SE}$ & 17years/NED & 1995 \\
\hline 1 & Menditti et al. [10] & $44 / \mathrm{M}$ & Lingual Mucosa & SE & 10years/NED & 1998 \\
\hline \multirow[t]{2}{*}{1} & Menditti et al. [10] & $34 / \mathrm{M}$ & Tongue & $\mathrm{SE}$ & 10years/NED & 1999 \\
\hline & $\begin{array}{l}\text { Fielman and } \\
\text { Morrow [13] }\end{array}$ & $11 / \mathrm{M}$ & Soft Palate & SE & 8M/NED & 1989 \\
\hline 1 & Srikanth et al. [14] & $27 / M$ & $\begin{array}{l}\text { Subcutaneous- } \\
\text { cheek }\end{array}$ & SE & & 2014 \\
\hline 1 & Rullo et al. [16] & $9 \mathrm{~m} / \mathrm{M}$ & Tongue & SE & $*$ & 2012 \\
\hline 2 & $\begin{array}{l}\text { Hoffman and } \\
\text { Martinez [17] }\end{array}$ & $8 / \mathrm{M}$ & Buccal Mucosa & $\mathrm{SE}$ & 14M/NED & 1981 \\
\hline 1 & $\begin{array}{c}\text { Weerapradist and } \\
\text { Punyasingh [18] }\end{array}$ & $50 / \mathrm{F}$ & Retromolar area & $\mathrm{SE}$ & $*$ & 1984 \\
\hline 1 & Fletcher [19] & $45 / \mathrm{M}$ & Subcutaneous Face & SE & $*$ & 1990 \\
\hline 1 & Fletcher [19] & $31 / \mathrm{M}$ & Intramusular scalp & $\mathrm{SE}$ & $*$ & 1990 \\
\hline 1 & Fletcher [19] & $56 / \mathrm{M}$ & $\begin{array}{l}\text { Intramuscular } \\
\text { cheek }\end{array}$ & $\mathrm{SE}$ & * & 1990 \\
\hline 1 & $\begin{array}{l}\text { Alonso del and } \\
\text { Hayo et al. [20] }\end{array}$ & $68 / \mathrm{M}$ & Buccal Mucosa & $*$ & 12M/NED & 1976 \\
\hline 1 & $\begin{array}{c}\text { O'Brien and Stout } \\
\text { [23] }\end{array}$ & $50 / \mathrm{F}$ & Buccal Mucosa & $\mathrm{SE}$ & 24M/NED & 1964 \\
\hline 1 & $\begin{array}{c}\text { Hillis and Beasley } \\
{[24]}\end{array}$ & $52 / \mathrm{M}$ & Internal Left Cheek & $\mathrm{SE}$ & & 1975 \\
\hline 1 & $\begin{array}{l}\text { Thompson and } \\
\text { Shear [25] }\end{array}$ & $49 / \mathrm{F}$ & Retromolar area & $\mathrm{SE}$ & 10M/NED & 1984 \\
\hline 1 & $\begin{array}{l}\text { Thompson and } \\
\text { Shear [25] }\end{array}$ & $36 / \mathrm{M}$ & Maxillary Gingiva & $\mathrm{SE}$ & 12M/NED & 1984 \\
\hline 1 & $\begin{array}{l}\text { Thompson and } \\
\text { Shear [25] }\end{array}$ & $44 / F$ & Base of Tongue & $\mathrm{SE}$ & $\begin{array}{c}\text { 11years and 7M/ } \\
\text { NED }\end{array}$ & 1984 \\
\hline 1 & $\begin{array}{l}\text { Thompson and } \\
\text { Shear [25] }\end{array}$ & $49 / \mathrm{F}$ & Palate & $\mathrm{SE}$ & 7M/NED & 1984 \\
\hline
\end{tabular}




\section{Advances in Dentistry \& Oral Health}

\begin{tabular}{|c|c|c|c|c|c|c|}
\hline 1 & $\begin{array}{l}\text { Thompson and } \\
\text { Shear [25] }\end{array}$ & $17 / \mathrm{M}$ & Buccal Mucosa & $\mathrm{SE}$ & 7MNED & 1984 \\
\hline 1 & $\begin{array}{l}\text { Triantafyllou et } \\
\text { al. [26] }\end{array}$ & $70 / \mathrm{M}$ & $\begin{array}{l}\text { Tip of Dorsal } \\
\text { Tongue }\end{array}$ & SE & 7Years/NED & 1985 \\
\hline 1 & $\begin{array}{c}\text { McLeod and Jones } \\
{[27]}\end{array}$ & $22 / \mathrm{F}$ & Lower Lip & $\mathrm{SE}$ & & \\
\hline 1 & Hong et al. [28] & $74 / F$ & Floor of mouth & SE & 9M/NED & 1999 \\
\hline 1 & $\begin{array}{c}\text { Ide and Kusama } \\
{[29]}\end{array}$ & $50 / \mathrm{F}$ & $\begin{array}{l}\text { Mandibular } \\
\text { Gingiva }\end{array}$ & $\mathrm{SE}$ & 20years/NED & 2002 \\
\hline 1 & Yamada et al. [30] & $6 \mathrm{~m} / \mathrm{M}$ & Upper lip & SE & $*$ & 2002 \\
\hline 1 & Alves et al. [31] & $26 / \mathrm{F}$ & Buccal Mucosa & SE & 24M/NED & 2003 \\
\hline 1 & Hidaka et al. [32] & $2 \mathrm{y} 8 \mathrm{~m} / \mathrm{M}$ & Maxillary Gingiva & SE & 4M/NED & 2005 \\
\hline 1 & $\begin{array}{c}\text { Toyohara et al. } \\
\text { [33] }\end{array}$ & $76 / \mathrm{F}$ & Upper Lip & $\mathrm{SE}$ & 4years/NED & 2008 \\
\hline 1 & Lee et al. [34] & $41 / \mathrm{F}$ & Upper lip & $\mathrm{SE}$ & $*$ & 2010 \\
\hline 1 & Bage et al. [35] & $59 / \mathrm{F}$ & Right Cheek & SE & 14M/NED & 2010 \\
\hline 1 & $\begin{array}{l}\text { Lopez Lornet et } \\
\text { al. [36] }\end{array}$ & $8 / \mathrm{F}$ & Dorsum of Tongue & $\mathrm{SE}$ & * & 2011 \\
\hline 1 & Bindhu et al. [37] & $20 / \mathrm{F}$ & Hard Palate & SE & $*$ & 2012 \\
\hline 1 & Caldeira et al. [38] & $29 / \mathrm{F}$ & Hard palate & SE & $*$ & 2012 \\
\hline 1 & Rajathi et al. [39] & $23 / \mathrm{M}$ & Gingiva & SE & $*$ & 2013 \\
\hline 1 & Priya et al. [40] & $30 / \mathrm{F}$ & Dorsum of Tongue & SE & 3Y/NED & 2013 \\
\hline 1 & Pandey et al. [41] & $26 / M$ & Tongue & SE & * & 2013 \\
\hline 1 & Shrier et al. [49] & Newborn (1 day) & Nasal Cavity & SE & $*$ & 1998 \\
\hline 1 & Dardo et al. [50] & $34 / \mathrm{M}$ & Tongue & SE & $12 \mathrm{M}$ & 1999 \\
\hline 1 & Dardo et al. [50] & $44 / \mathrm{M}$ & Floor of mouth & SE & 14M/NED & 1998 \\
\hline 1 & $\begin{array}{c}\text { Skoulakis et al. } \\
\text { [51] }\end{array}$ & 19/M & Cheek & SE & $*$ & 2007 \\
\hline 1 & Pia et al. [52] & $8 / \mathrm{F}$ & Tongue & SE & & 2011 \\
\hline 1 & Nur et al. [53] & $10 / \mathrm{F}$ & $\begin{array}{l}\text { Extrnal Auditoy } \\
\text { canal }\end{array}$ & SE & 12M/NED & 2012 \\
\hline 1 & $\begin{array}{c}\text { Himanshu et al. } \\
\text { [54] }\end{array}$ & $62 / F$ & Buccal Mucosa & SE & 12M/NED & 2012 \\
\hline 1 & $\begin{array}{c}\text { Narendra et al. } \\
\text { [55] }\end{array}$ & $26 / \mathrm{M}$ & Tongue & SE & * & 2013 \\
\hline 1 & Pradipta et al. [56] & $45 / \mathrm{M}$ & $\begin{array}{l}\text { Submandibular } \\
\text { space }\end{array}$ & SE & 14M/NED & 2013 \\
\hline
\end{tabular}

SE: Surgical excision; FU: Follow-up;NED:No evidence of disease.

Table 4: Review of cases of BFH of Hard tissues in chronological order.

\begin{tabular}{|c|c|c|c|c|c|c|}
\hline $\begin{array}{c}\text { No. of } \\
\text { cases }\end{array}$ & Authors & Age/Sex & Location & Treatment & $\begin{array}{c}\text { FU time/ } \\
\text { Recurrence }\end{array}$ & Year \\
\hline 1 & Saluja et al. [12] & $23 / \mathrm{F}$ & Maxilla & SE & $24 \mathrm{M} /$ NED & 2014 \\
\hline 1 & Shoor et al. [14] & $30 / \mathrm{F}$ & Posterior Mandible & SE & $24 \mathrm{M} / \mathrm{NED}$ & 2015 \\
\hline 1 & Cale et al. [42] & $13 / \mathrm{M}$ & Posterior Maxilla & SE & $14 \mathrm{M} / \mathrm{NED}$ & 1983 \\
\hline 1 & Ertas et al. [43] & $13 / \mathrm{F}$ & Anterior Mandible & SE & $12 \mathrm{M} /$ NED & 2003 \\
\hline 1 & Hio et al. [44] & $42 / \mathrm{M}$ & Posterior Mandible & SE & 2004 \\
\hline 1 & Kishino et al. [45] & $49 / \mathrm{M}$ & Posterior Mandible & SE & $7 \mathrm{M} /$ NED & 2005 \\
\hline 1 & Katagiri et al. [46] & $48 / \mathrm{M}$ & Mandible-Condyle & SE & $12 \mathrm{M} /$ NED & 2008 \\
\hline 1 & Wagner et al. [47] & $41 / \mathrm{M}$ & Posterior Mandible & Piezoelectric assisted SE & $10 \mathrm{M} / \mathrm{NED}$ & 2011 \\
\hline 1 & Gupta et al. [48] & $24 / \mathrm{F}$ & Posterior Mandible & SE & $12 \mathrm{M} /$ NED & 2011 \\
\hline
\end{tabular}


We have carried out an exhaustive research of all the Oral Benign Fibrous Histiocytoma tumours documented in literature since 1961-2015 and we have tabulated the findings received (Table 3 \& 4).

\section{Conclusion}

To the best of our understanding, oral BFH tumours have excellent prognosis and lesser chances of recurrences on management with complete surgical en bloc resection. These benign tumours show good loco regional behaviour post- surgical management. Chemo or Radiotherapy currently has no role in their management.

Thorough clinical history,prompt and correct diagnosis, complete excision with pathological margin clearance and regular follow up is imperative in the management of BFH. However complete understanding, knowledge and awareness of the innate behaviour of these tumours is an indispensable trait in a Head and Neck Surgeon.

\section{References}

1. Kumar P, Umesh, Rathi T, Jain V (2016) Benign Fibrous Histiocytoma: A Rare Case Report and Literature Review, J Oral Maxillofac Surg 15(1): 116-120.

2. Prisse LA, Jayasooriya PR, Mendis BR, Lombardi T (2015) Benign Fibrous Histiocytomas of the Oral Mucosa: Report on Three Cases and Review of the Literature Dermatopathology (Basel) 2(2): 52-60.

3. Neville BW, Damm DD, Allen CM, Bouquot JE (2001) Oral and Maxillofacial Pathology, $\left(2^{\text {nd }} e d n\right)$, Philadelphia, WB Saunders, Elsevier, USA pp. 368-369.

4. Weiss S, Goldblum J (2008) Enzinger and Weiss's Soft Tissue Tumors, ( $5^{\text {th }}$ edn) St Louis, Mobsy, pp. 331-348.

5. Giovani P, Patrikidou A, Ntomouchtsis A, Meditskou S, Thuau H, et al. (2010) Benign fibrous histiocytoma of the buccal mucosa: case report and literature review. Case Rep Med 2010: 306148.

6. Priya NS, Rao K, Umadevi HS, Smith T (2013) Benign fibrous histiocytoma of the tongue. Indian J Dent Res 24(5): 635-638.

7. F Femiano, C Scully, G Laino, G Battista (2001) Benign fibrous histiocytoma (BHF) of the cheek: CD 68-KP1 positivity. Oral Oncology 37(8): 673-675.

8. Gray PB, Miller AS, Loftus MJ (1992) Benign fibrous histiocytoma of the oral/perioral regions: Report of a case and review of 17 additional cases. J Oral Maxillofac Surg 50(11): 1239-1242.

9. Bielamowicz S, Dauer MS, Chang B, Zimmerman MC (1995) Noncutaneous benign fibrous histiocytoma of the head and neck. Otolaryngol Head Neck Surg 113(1): 140-146.

10. Menditti D, Laino L, Mezzogiorno A, Sava S, Bianchi A, et al. (2009) Oral benign fibrous histiocytoma: two case reports. Cases J 2: 9343.

11. Del Hoyo JA, Contreras F, Gonzales (1976) FD: Fibro-histiocytome d e la cavité buccale. Rev Stomatol Chir Maxillofac 77: 481-483.

12. Saluja H, Kasat VO, Rudagi BM, Dehane V, Kalburge JV, et al. (2014) Benign fibrous histiocytoma of the maxilla: a case report and review of literature. Indian J Dent Res 25(1):115-118.

13. Fieldman RJ, Morrow TA (1989) Fibrous histiocytoma of the soft palate. Int J Pediat Otorhinolaryngol 18: 171-179.
14. Srikanth D, Devi V, Polishetty V, Singh D (2015) Subcutaneous Benign Fibrous Histiocytoma: Rare Presentation on Cheek-Case Report and Review of Literature. J Maxillofac Oral Surg 15(Suppl 2): 282-286.

15. Vuity D, Bogdan S, Csurgay K, Sapi Z, Nemeth Z (2013) Pathol Oncol Res 19(4): 605-609.

16. Rullo R, Ferraraccio F, Serpico R, Addabbo F, Mazzarella N, et al. (2012) Oral fibrous histiocytoma and its angiomatoid variant. J Craniomaxillofac Surg 40(5): 435-438.

17. Hoffman S, Martinez MG (1981) Fibrous histiocytoma of the oral mucosa 52: 277-283.

18. Weerapradist W, Punyasingh J (1984) Fibrous histiocytoma: report of a case of the oral mucosa J Dent Assoc Thai 34: 263-269.

19. Shrier DA, Wang AR, Patel U, Monajati A, Chess P, et al. (1998) Benign fibrous histiocytoma of the nasal cavity in a newborn: MR and CT findings. Am J Neuroradiol 19(6): 1166-1168.

20. Lisle DA, Monsour PA, Maskiell CD (2008) Imaginng of craniofacial fibrous dysplasia. J Med Imagin Radiat Oncol 52(4): 325-332.

21. E Calonje, T Mentzel, CDM Fletcher (1994) Cellularbenign fibrous histiocytoma: clinicopathologic analysis of 74 cases of a distinctive variant of cutaneous fibrous histiocytoma with frequent recurrence. Am J Surg Pathol 18(7): 668-676.

22. Bali A, Singh MP, Padmavathi, Khorate M, Ahmed J (2010) Malignant Fibrous Histiocytoma - An Unusual Transformation from Benign to Malignant. J Cancer Sci Ther 2: 053-057.

23. O'Brien JE, Stout AP (1964) Malignant Fibrous Xanthomas. Cancer 17: $1445-1455$

24. Hillis RE, Beasley JD (1975) Fibrous histiocytoma of the lip: rep ort of a case. J Oral Med 30: 81-83.

25. Thompson SH, Shear M (1984) Fibrous histiocytomas of the oral and maxillofacial regions. J Oral Pathol 13(3): 282-294.

26. Triantafyllou AG, Sklavounou AD, Laskaris GG (1985) Benign fibrous histiocytoma of the oral mucosa. J Oral Med 40: 36-38.

27. McLeod SPR, Jones JL (1992) Fibrous histiocytoma of the lip secondary to trauma: report of a case. J Oral Maxillofac Surg 50(10): 1091-1093.

28. Hong KH, Kim YK, Park JK (1999) Benign fibrous histiocytoma of the floor of the mouth. J Otolaryngol Head Neck Surg 121: 330-333.

29. Ide F, Kusama K (2002) Benign fibrous histiocytoma: an additional case richly endowed with factor XIIIa cells. Oral Oncol 38(3): 321-322.

30. Yamada H, Ishii H, Kondoh T, Seto K (2002) A case of benign fibrous histiocytoma of the upper lip in a 6-month-old infant. J Oral Maxillofac Surg 60(4): 451-454.

31. Alves FA, Vargas PA, Coelho Sicueira SA, Coletta RD, De Alemida OP (2003) Benign fibrous histiocytoma of the buccal mucosa: case report with immunohistochemical features. J Oral Maxillofac Surg 61(2): 269271.

32. Hidaka M, Yamashita A, Sakamoto K, Mukaisho K, Hattori T, et al. (2005) Benign fibrous histiocytoma occured in the alveolar mucosa accompanying with sialidosis type 2 in a Japanese infant. Oral Oncol 41(5): 253-258.

33. Toyohara Y, Hashitani S, Sakurai K, Takaoka K, Urade M (2008) Benign fibrous histiocytoma of the upper lip: a case report. Oral Science Internat 5(1): 65-68.

34. Lee HI, Lee JW, Han TY, Li K, Hong CK, et al. (2010) A case of dermatofibroma of the upper lip. Ann Dermatol 22: 333-336. 
35. Bage AM, Bylappa K, Kumar MV (2010) A rare case of fibrous histio cytoma of subepidermal soft tissue of cheek (buccal mucosa). Internet J Otorhinolaryngol 13: 11.

36. Lopez Jornet P, Camacho Alonso F, Gomez Garcia FJ (2011) Oral lesion of the dorsum of the tongue. J Can Dent Assoc 77: b117.

37. Bindhu PR, Padmakumar SK, Priya T, Jacob J (2012) A rare case of benign fibrous histiocytoma in the submucosal soft tissue of the hard palate: a case report and review of the literature. Oral Maxillofac Pathol J $3: 215-217$.

38. Caldeira PC, Ribeiro DC, Almeida OP, Mesquita RA, Do Carom MAV (2012) Tumour of the hard palate. Oral Surg Oral Med Oral Pathol Oral Radiol 113(6): 722-727.

39. Rajathi P, Jacob M, Priyadarshani I, Sekar B (2013) Benign fibrous histiocytoma of the gingiva. J Pharm Bioallied Sci 5 (suppl 2): S166-S168.

40. Priya NS, Rao K, Umadevi HS, Smith T (2013) Benign fibrous histiocytoma of the tongue. Indian J Dent Res 24: 635-638.

41. Pandey NK, Sharma SK, Banerjee S (2013) A rare case of fibrous histiocytoma of the tongue. Indian J Surg 75(suppl 1): 1-5.

42. Cale AE, Freedman PD, Kerpel SM, Lumerman H (1989) Benign fibrous histiocytoma of the maxilla. Oral Surg Oral Med Oral Pathol 68(4): 444450.

43. Ertas U, Buyukkurt MC, Cicek Y (2003) Benign fibrous histiocytoma: report of case. J Contemp Dent Pract 4(2): 74-79.

44. Heo MS, Cho HJ, Kwon KJ, Lee SS, Choi SC (2004) Benign fibrous histiocytoma in the mandible. Oral Surg Oral Med Oral Pathol Oral Radiol Endod 97(2): 276-280.

45. Kishino M, Murakami S, Toyosawa S, Nakatami A, Ogawa Y, Ishida T, et al (2005) Benign fibrous histiocytoma of the mandible. J Oral Pathol Med 34(3): 190-192.

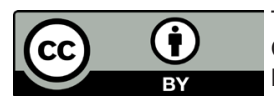

This work is licensed under Creative Commons Attribution 4.0 License

DOI: 10.19080/ADOH.2018.08.555748
46. Katagiri W, Nakazawa M, Kishino M (2008) Benign fibrous histiocytoma in the condylar process of the mandible: case report. Br J Oral Maxillofac Surg 46(1): e1-e2.

47. Wagner ME, Rana M, Traenkenschuh W, Kokemueller H, Eckardt AM, et al. (2011) Piezoelectric-assisted removal of a benign fibrous histiocytoma of the mandible: an innovative technique for prevention of dentoalveolar nerve injury. Head Face Med 7:20.

48. Gupta P, Godhi SS, Kukreja P, Bhatnagar S, Lall AB, et al. (2011) Fibrous histiocytoma of the mandible-a case report. J Indian Dent Assoc 5: $994-$ 995.

49. Shrier DA, Wang AR, Patel U, Monajati A, Chess P, et al. (1998) Benign fibrous histiocytoma of the nasal cavity in a newborn: MR and CT findings. Am J Neuroradiol 19(6): 1166-1168.

50. Dardo M, Luigi L, Antonio M, Sara S, Alexander B, et al. (2009) Oral benign fibrous histiocytoma: two case reports. Cases J 2: 9343.

51. Skoulakis CE, Papadakis CE, Datseris GE, Drivas EI, Kyrmizakis DE, et al. (2007) Subcutaneous benign fibrous histiocytoma of the cheek. Case report and review of the literature. Acta Otorhinolaryngol Ital 27(2): 90-93.

52. Pı'a LJ, Fabio C, Francisco J (2011) Oral lesion on dorsum of tongue. J Can Dent Assoc 77: b117.

53. Nur HA, Bee S, Fauzah A, Lokman S (2012) Benign fibrous histiocytoma of the external auditory canal: case report and literature review. Bangladesh J Otorhinolaryngol 18(1): 77-80.

54. Himanshu S, Sarwar A, Sonal U, Harsh Y, Paankhi L, et al. (2013) Benign fibrous histiocytoma of buccal mucosa. J Dental Sci Oral Rehabil p. 3840 .

55. Narendra K, Sushant K, Sandip B (2013) A rare case of fibrous histiocytic tumor of the tongue. Indian J Surg 75(Suppl 1): S1-S5.

56. Pradipta K, Gopalakrishnan S, Sivaraman G (2013) Benign fibrous histiocytoma of submandibular space. Pak J Otolaryngol 29: 96-98.

\section{Your next submission with Juniper Publishers will reach you the below assets}

- Quality Editorial service

- Swift Peer Review

- Reprints availability

- E-prints Service

- Manuscript Podcast for convenient understanding

- Global attainment for your research

- Manuscript accessibility in different formats

( Pdf, E-pub, Full Text, Audio)

- Unceasing customer service

Track the below URL for one-step submission https://juniperpublishers.com/online-submission.php 